\title{
Two-vessel chronic total occlusion. Complete percutaneous revascularisation
}

\author{
Artur Dębski, Paweł Tyczyński, Maksymilian P. Opolski, Mariusz Kłopotowski, Maciej A. Karcz, \\ Adam Witkowski
}

Department of Interventional Cardiology and Angiology, Institute of Cardiology, Warsaw, Poland

Postep Kardiol Inter 2014; 10, 4 (38): 320-322

DOI: $10.5114 /$ pwki.2014.46779

\begin{abstract}
A bstract
Little is known about the success rate of second attempts to open chronic total occlusions. Two-vessel occlusion makes the procedure is even more challenging. Thus, embarking on complete percutaneous revascularization of such lesions requires adequate experience, especially after first unsuccessful attempt. We present a case of a 52-year-old male patient in whom successful percutaneous opening of two chronic coronary at staged procedure was performed.
\end{abstract}

Key words: multiple chronic total occlusion, percutaneous coronary intervention.

\section{Case report}

A 52-year-old-patient with a history of previous myocardial infarction was scheduled for further diagnosis due to recurrent angina class CCS II/III. The echocardiography showed mildly impaired left ventricle systolic function, ejection fraction of $50 \%$, and akinesia limited to mid segments of the inferior and lateral wall. Coronary angiography revealed chronic total occlusion (CTO) of both the left anterior descending coronary artery (LAD) and the left circumflex coronary artery (LCX) (Figure $1 \mathrm{~A}$ ) and non-significant lesions in the right coronary artery. The patient definitely refused the surgical treatment of coronary lesions. Thus, an attempt to open the LCX was carried out in a secondary center. Radial access was used to cannulate the left coronary artery with a JL 4.0 guide catheter. The LCx occlusion was crossed with Pilot 50 wire (Abbott Vasc, Santa Clara, USA). However, a $1.5 \mathrm{~mm}$ rapid exchange balloon was unable to follow the wire past the occlusion. The procedure was stopped and the patient has been referred to our department for further treatment.

At the first step, successful opening of the LAD with Sion Blue wire (Asahi Intecc, Japan) in the direction of diagonal branch was done. After unsuccessful negotiation of the distal LAD segment with Fielder XT wire (Asahi Intecc, Japan), Confianza wire (Asahi Intecc, Japan) was ad- vanced via microcatheter Corsair (Asahi Intecc, Japan) and crossed the occlusion. After predilatation, a $3.0 \times 38 \mathrm{~mm}$ everolimus eluting stent was implanted (Figure $1 \mathrm{~B}$ ).

At staged procedure an AL2 guide catheter was used to cannulate the left coronary artery ostium. Fielder FC (Asahi Intecc, Japan) wire successfully negotiated through the LCx occlusion. Then a $1.2 \mathrm{~mm}$ MiniTrek Over The Wire balloon (Abbott Vasc, Santa Clara, USA) crossed the lesion. After predilatation, Sion Blue was advanced into the marginal branch. Finally, a $2.5 \times 28 \mathrm{~mm}$ everolimus eluting stent at 14 atm was implanted (Figure $1 \mathrm{C}, \mathrm{D})$.

For both coronaries TIMI 3 flow was noted at the end of the procedure. The total fluoroscopic time for both procedures was $60 \mathrm{~min}$ (2607 mGy). For both approaches, the femoral artery was used as a vascular access. The postprocedural course was uneventful.

\section{Discussion}

Multiple CTO is a rare finding as compared to single CTO and the prognosis with conservative treatment is poorer [1]. Surgical treatment is usually an attractive option for such patients unless lack of patient consent or contraindications necessitate a percutaneous approach. Successful percutaneous coronary intervention (PCI) of all CTOs is associated with increased survival,

\section{Corresponding author:}

Paweł Tyczyński MD, PhD, Department of Interventional Cardiology and Angiology, Institute of Cardiology, 42 Alpejska St, 04-628 Warsaw, Poland, phone: +48 696 091079, e-mail: medykpol@wp.pl

Received: 9.07.2014, accepted: 29.09.2014. 

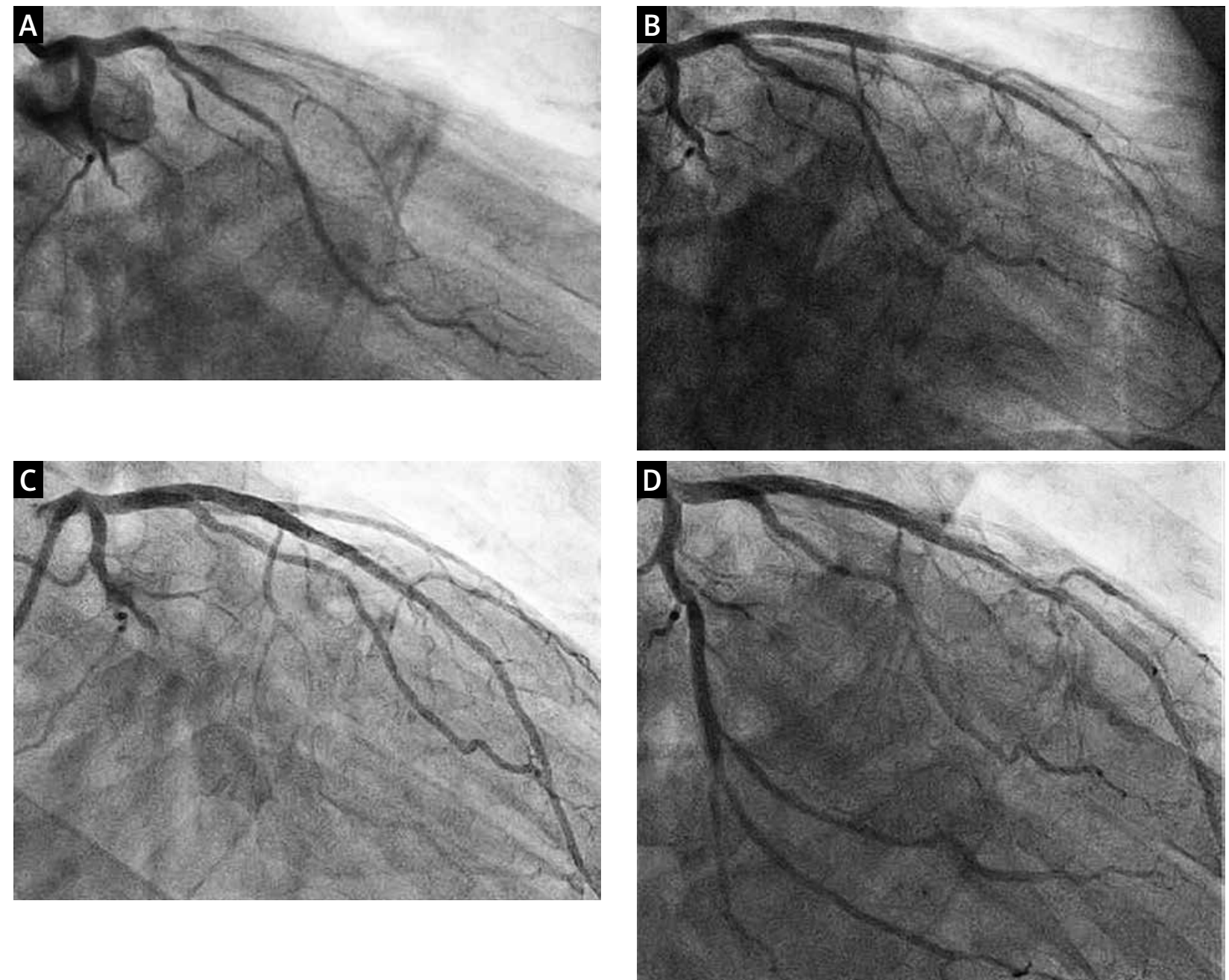

Figure 1. A - CTO of the LAD and the LCX. B - LAD after opening and implantation of DES. C - CTO of LCX. D - LCX after opening and implantation of DES

CTO - chronic total occlusion, LAD - left anterior descending coronary artery, LCX - left circumflex coronary artery, DES - drug eluting stent

and complete of revascularisation is a strong predictor of survival [2]. However, scarce data are available regarding complete percutaneous revascularisation of multiple CTO.

Furthermore a failed attempt to open CTO is a predictor of lower success rate of a second approach. In the J-CTO registry the success rate of first CTO attempt was as high as $88 \%$. Repeat CTO attempt, however, was associated with only $65 \%$ success rate [3]. Wire failure to cross over the occlusion is the most common reason of unsuccessful CTO PCI. Ten-twenty percent of the CTO PCI failures are due to balloon un-crossable lesions. Methods to deal with such problem start with simple technical tips, like better guide catheter support and deep sitting or buddy wire. Radial access, which was used for the first attempt to open the CTO in our patient, may not provide sufficient back-up support. Although the femoral access is preferable for CTO $\mathrm{PCl}$, single reports stress the value of a radial approach [4]. The use of more advanced tech- niques requires adequate experience and often referral to a higher volume operator (as in our case). These alternatives include "mother-and-child" technique, anchoring, Tornus catheter, etc.

Finally due to the nature of CTO procedures, a staged approach is the logical choice. This lowers the risk of contrast-induced nephropathy (CIN) and possible X-ray related skin injury. In the aforementioned J-CTO registry, the total fluoroscopic time per patient was $45 \mathrm{~min}$, the contrast volume was $293 \mathrm{ml}$, and the frequency of CIN was $1 \%$. However, another Asian group reported the CIN rate at 5\% [5]. The procedural outcomes of CTO PCI among the operators of the EuroCTO club are comparable to those reported in the Japanese registry [6].

\section{References}

1. Bataille Y, Déry JP, Larose E, et al. Impact of percutaneous coronary intervention for chronic total occlusion with multivessel disease. Heart 2012; 98: 1732-7. 
2. Danzi GB, Valenti R, Migliorini A, et al. Percutaneous coronary intervention for multiple chronic total occlusions. Am J Cardiol 2013; 112: 1849-53.

3. Morino Y, Kimura T, Hayashi Y, et al. J-CTO Registry Investigators. In-hospital outcomes of contemporary percutaneous coronary intervention in patients with chronic total occlusion insights from the J-CTO Registry (Multicenter CTO Registry in Japan). JACC Cardiovasc Interv 2010; 3: 143-51.

4. Heuser R, Lassetter J. Successful two-vessel CTO intervention: importance of the radial approach. Vascular Disease Management. Blog 2010.

5. Lin YS, Fang HY, Hussein H, et al. Predictors of contrast-induced nephropathy in chronic total occlusion percutaneous coronary intervention. Eurolntervention 2014; 9: 1173-80.

6. Galassi AR, Tomasello SD, Reifart N, et al. In-hospital outcomes of percutaneous coronary intervention in patients with chronic total occlusion: insights from the ERCTO (European Registry of Chronic Total Occlusion) registry. Eurolntervention 2011; 7: 472-9. 